\title{
The Effect of Informational Support on Satisfaction of Family Caregivers of Patients with Hemiplegic Stroke from Receiving Information
}

\author{
Azim Azizi', Mahnaz Khatiban², Zhale Mollai ${ }^{3^{*}}$, Younes Mohammadi ${ }^{4}$
}

1. Assistant Professor, Chronic Diseases (Home Care) Research Centre, Hamadan University of Medical Sciences, Hamadan, Iran

2. Associate Professor, Department of Medical Surgical Nursing, School of Nursing and Midwifery, Hamadan University of Medical Sciences, Hamadan, Iran

3. MSc. Student, Department of Critical Care Nursing, Student Research Committee, School of Nursing and Midwifery, Hamadan University of Medical Sciences, Hamadan, Iran

4. Assistant Professor, Department of Epidemiology, School of Health, Hamadan University of Medical Sciences, Hamadan, Iran

\section{Article Info \\ Received: $\quad 2017 / 11 / 25$ \\ Accepted: $\quad$ 2018/01/16 \\ Published Online 2018/03/16}

DOI:

10.30699/sjhnmf.26.4.211

Original Article

Use your device to scan and read the article online

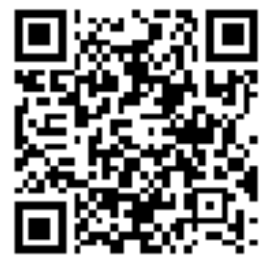

\section{Abstract}

Introduction: Satisfaction of patients' families is one of the criteria for assessing the quality of nurses' performance. Considering the low satisfaction of stroke patient's families in received information, the present study was done to determine effect of informational support on satisfaction of family caregivers of patients with hemiplegic stroke from received Information.

Methods: In this semi-experimental study, 78 family caregivers of patients with hemiplegic stroke admitted to the neurology department of Farshchian (Sina) Hospital of Hamadan (2017), were selected by Convenience sampling, and were divided continuously into two groups: control $(n=38)$ and intervention $(n=40)$. The research intervention was conducted from third to eleventh day of hospitalization. Information was provided about the department, equipment, patient status, home care individually and as a group method. The control group received routine care. Satisfaction of family caregivers was measured before and after the intervention in both groups. The data collecting tool was a questionnaire of demographic characteristics of the caregiver and patient, a researcher-made questionnaire to assess the satisfaction analyzing the received information. Data were analyzed using SPSS16.

Results: Groups were similar in terms of demographic variables. There was a significant difference between before (79.15 \pm 21$)$ and after (121.4 \pm 9.93$)$ intervention at satisfaction scores in intervention group, and between the two groups (intervention and control) after intervention $(P<0.001)$.

Conclusion: Informational support is effective on increasing the satisfaction of family caregivers of patients with stroke. Therefore, informational support is recommended as an important nursing intervention.

Keywords: Stroke, Access to Information, Patient Satisfaction, Caregivers
Zhale Mollai, MSc. Student, Department of Critical Care Nursing, Student Research Committee, School of Nursing and Midwifery, Hamadan University of Medical Sciences, Hamadan, Iran Email: Zhanet2811@gmail.com

Copyright $($ C 2018, Sci J Hamadan Nurs Midwifery Fac. This is an open-access article distributed under the terms of the Creative Commons Attribution-noncommercial 4.0 International License which permits copy and redistribute the material just in noncommercial usages, provided the original work is properly cited.

How to Cite This Article:

Azizi A, Khatiban M, Mollai Z, Mohammadi Y. The Effect of Informational Support on Satisfaction of Family Caregivers of Patients With Hemiplegic Stroke from Receiving Information . Sci J Hamadan Nurs Midwifery Fac. 2018; 26 (4) :211-218 


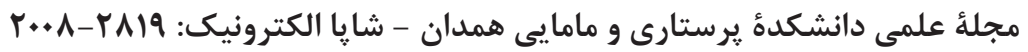

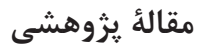

تأثير حمايت اطلاعاتى بر احساس رضايت مراقبان خانوادتى بيماران مبتلا به سكتهُ مغزى همى يلزيك اطنافي از دريافت اطلاعات

\section{عظيم عزيزى'، مهناز خطيبان'، زاله ملايى"'، يونس محمدى"}

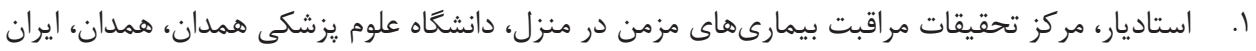

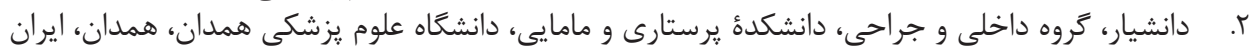

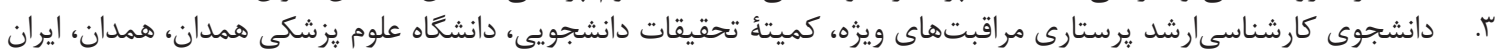

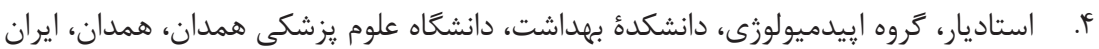

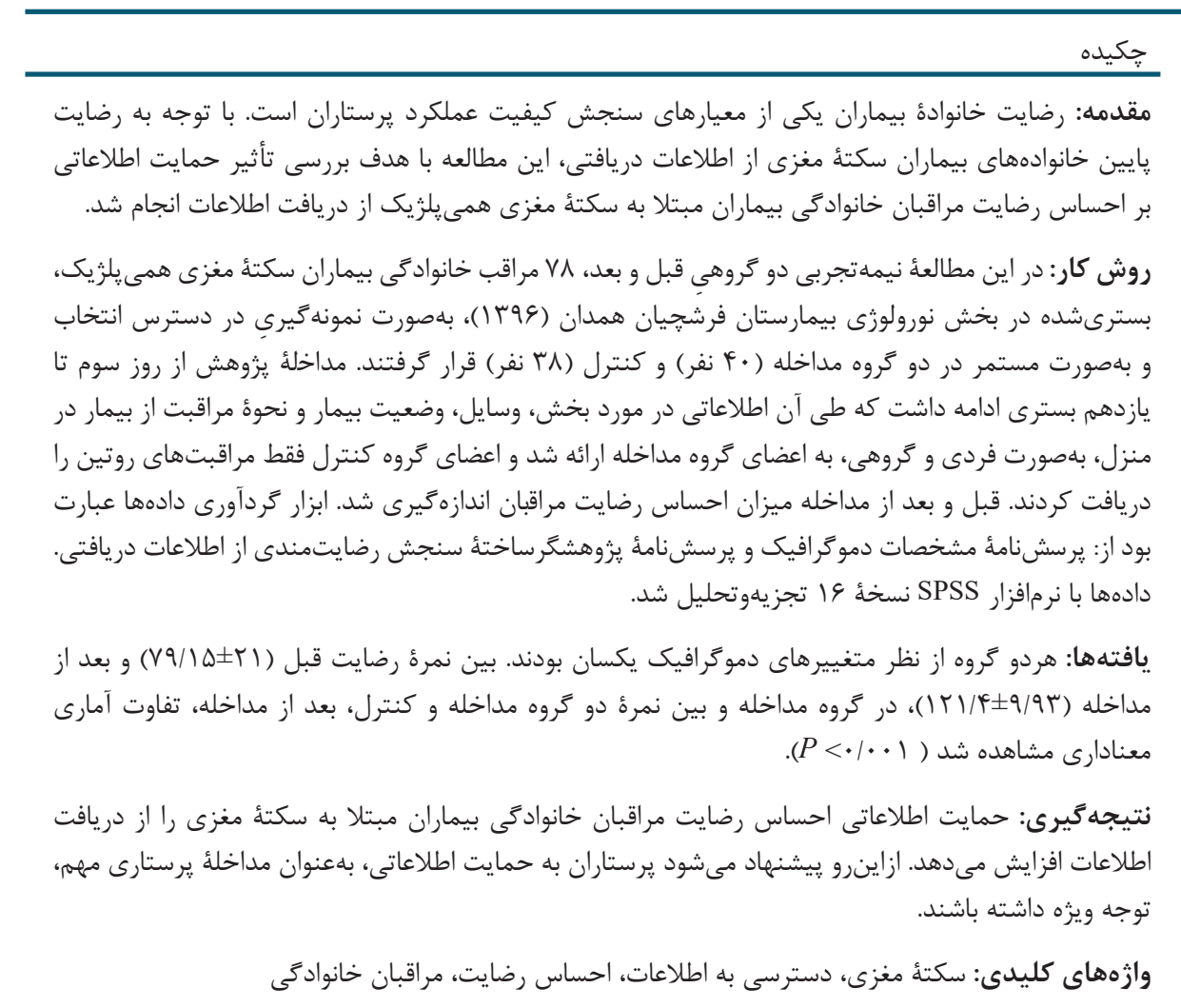

\begin{tabular}{|c|}
\hline اطلاعات مقاله \\
\hline $\mid r q 9 / 9 / \cdot r$ \\
\hline 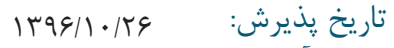 \\
\hline انتشار آنلاين: \\
\hline نويسندهُ مسئول: \\
\hline 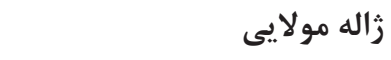 \\
\hline دانشجوى كارشناسىارشد \\
\hline يرستارى مراقبتهاى ويزه، عضو \\
\hline كميتأ تحقيقات دانشجويى، \\
\hline دانشخاه علوم يزشكى همدان، \\
\hline همدان، ايران \\
\hline يست الكترونيك \\
\hline
\end{tabular}

Zhanet2811@gmail.com
مبتلا به سكتأ مغزى نشان مى مدهد علىرغم رضايت عمومى إنى

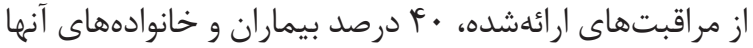

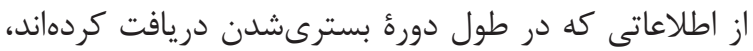

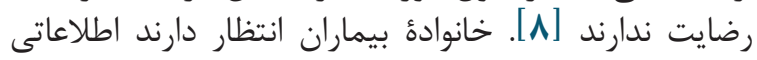

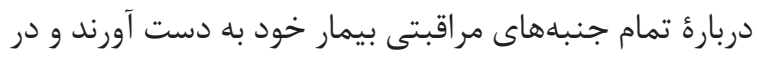

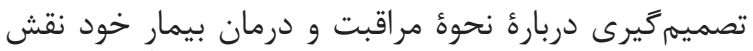

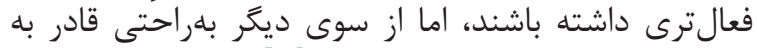

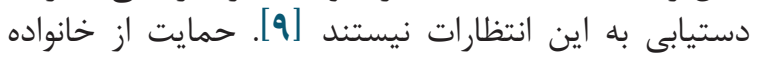

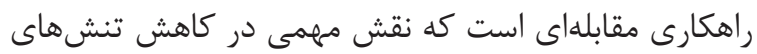

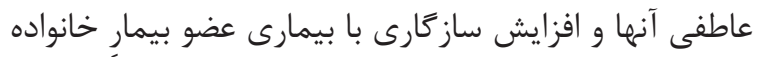

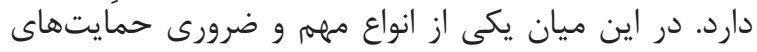

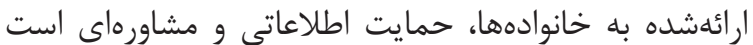

.[1.]
رضايتمندى، امروزه در مراقبتهاى يرستارى و يزشكى

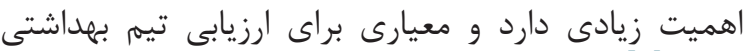

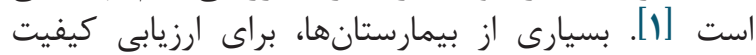

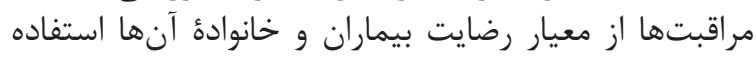

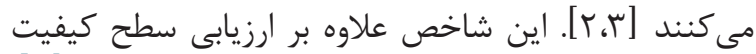

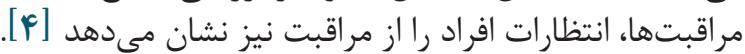

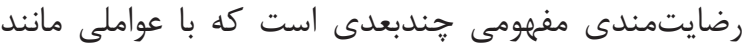

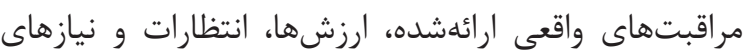

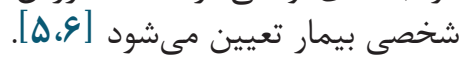

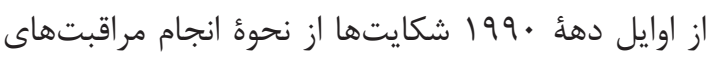

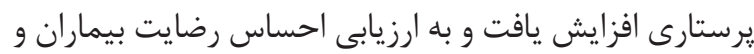

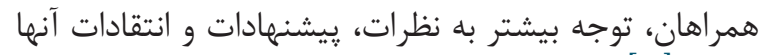

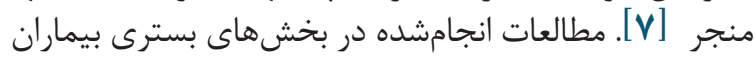


مغزى همى يلزيك انجام شد.

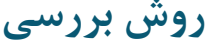

اين يزوهش مطالعهاى نيمهتجربى، همراه با طرح

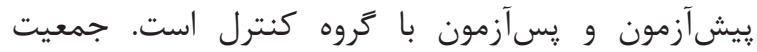

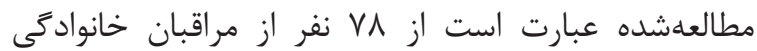

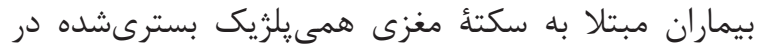

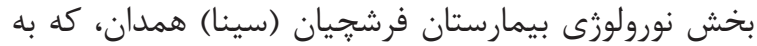

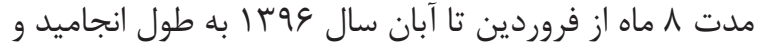

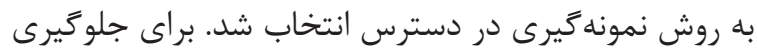

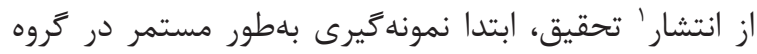

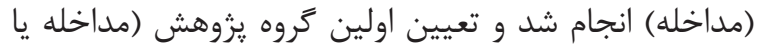

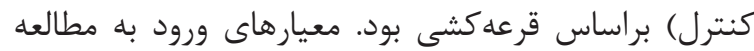

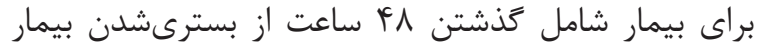

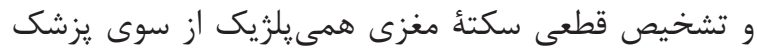

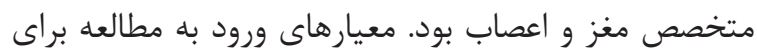

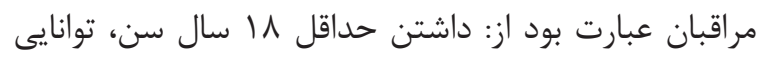

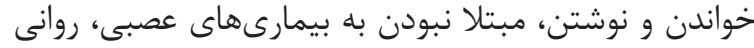

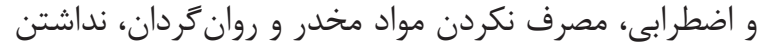

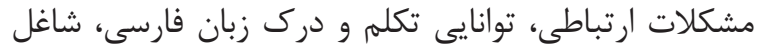

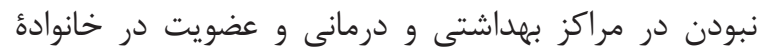

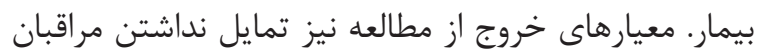

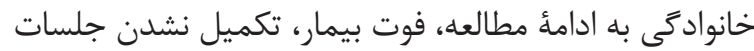

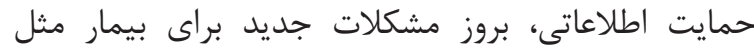

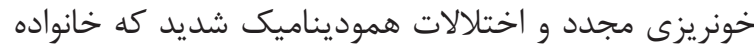

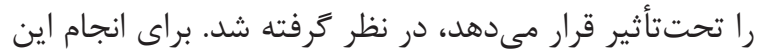

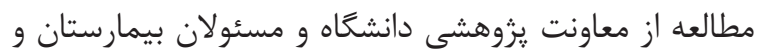

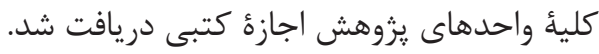

مداخلئ يزوهش بلهصورت حمايت اطلاعاتى مراقبان

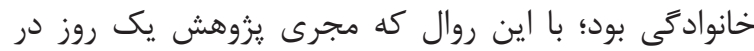

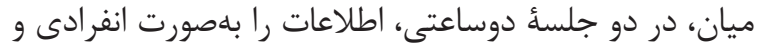

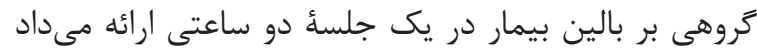

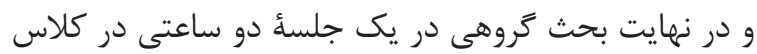

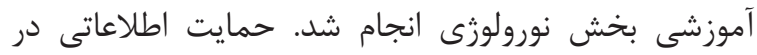

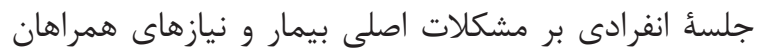

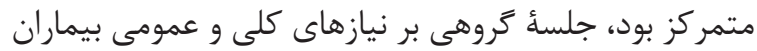

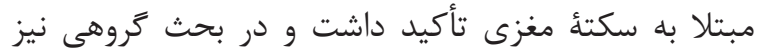

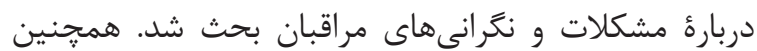

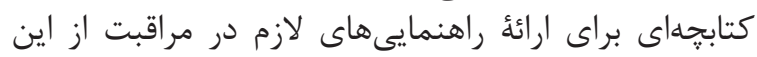

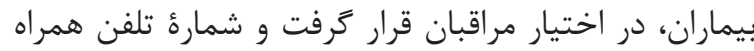

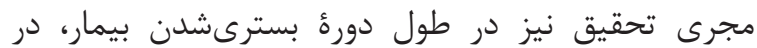

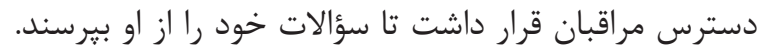

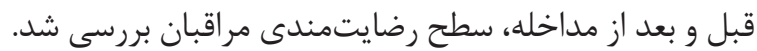

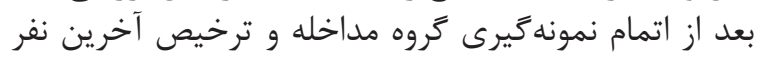

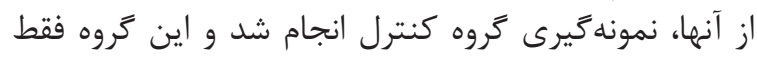

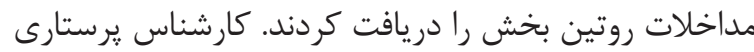

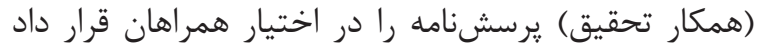

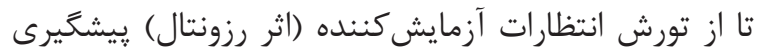

1. Diffusion

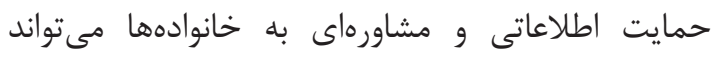

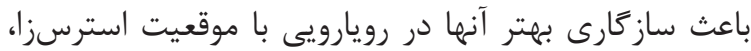

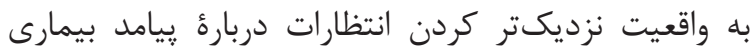

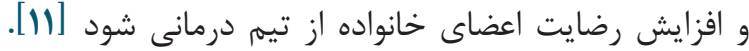

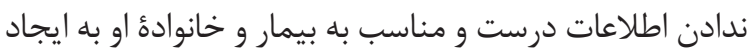

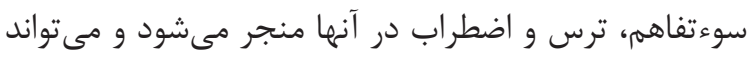

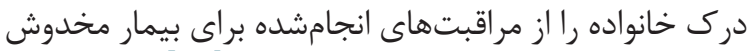

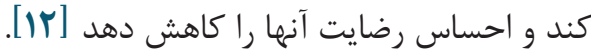

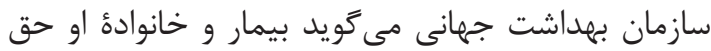

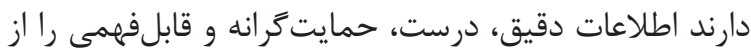

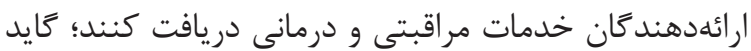

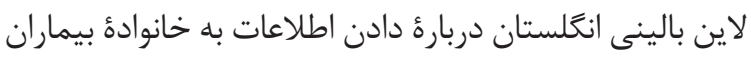

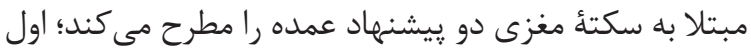

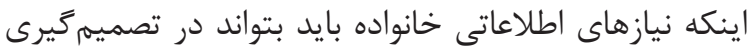

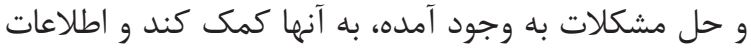

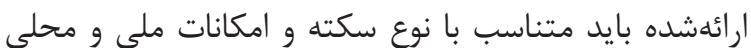

باشد [9].

مطالعة Drummond و همكاران نشان داد داد اطلاعاتى

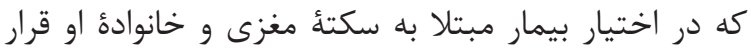

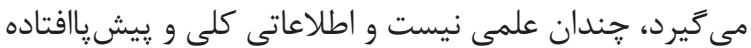

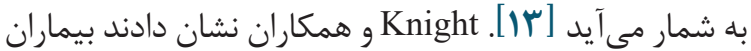

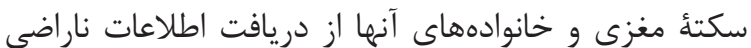

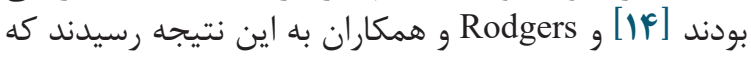

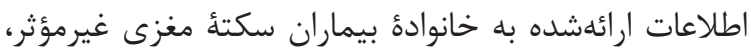

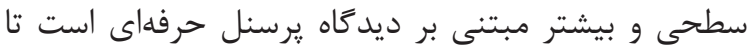

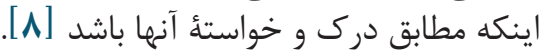

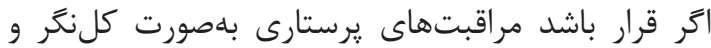

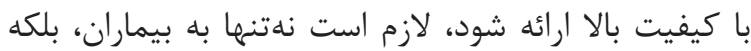

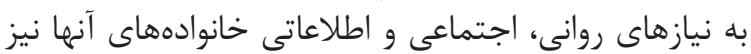

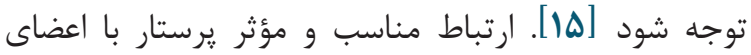

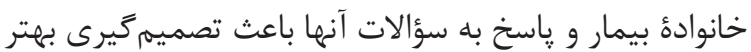

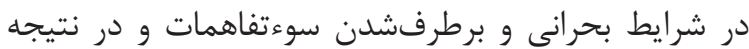

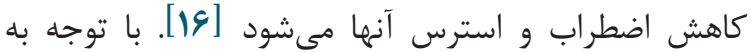

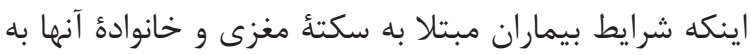

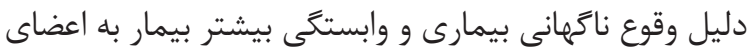

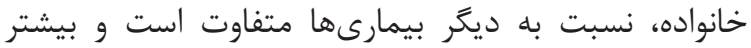

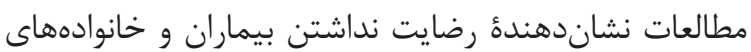

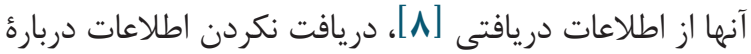

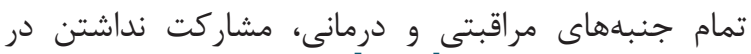

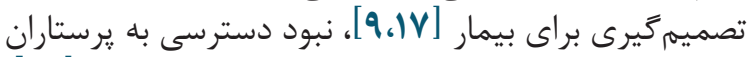

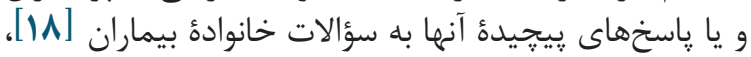

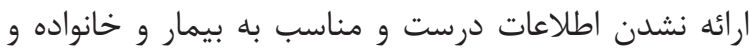

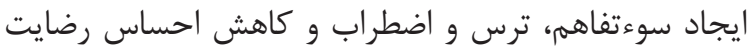

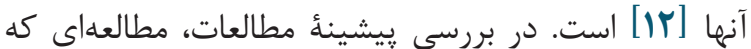

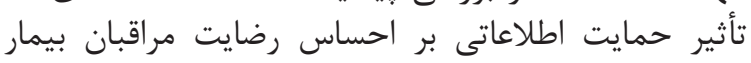

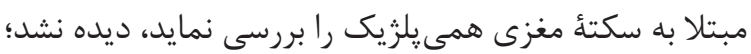

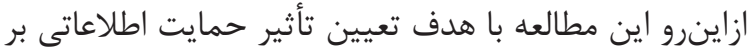

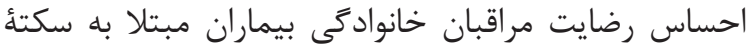




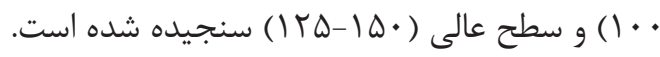

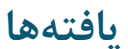

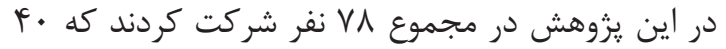

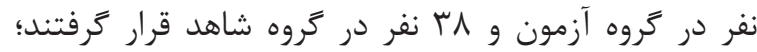

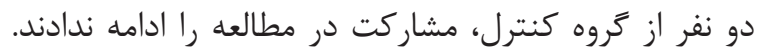

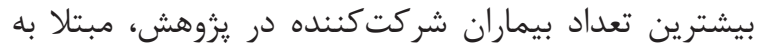

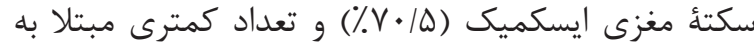

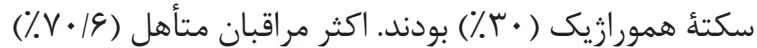

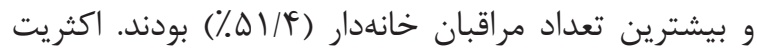

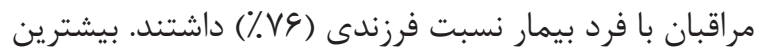
تعداد مراقبان شركت كننده در يزوهش زن فرئ (

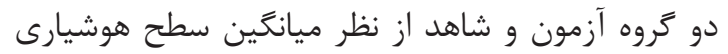
بيمار (I) (P) (P)

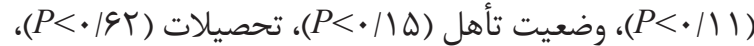

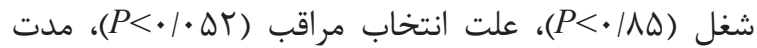

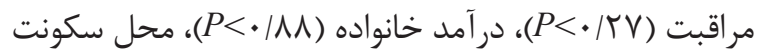

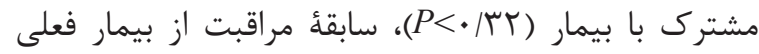
و أن

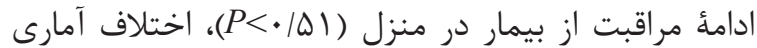
معنى دارى نداشتند و از نظر عوامل يادشده همخن بودند. مقايسٔ نمره رضايتمندى از اطلاعات دريافتى قبل و بعدي

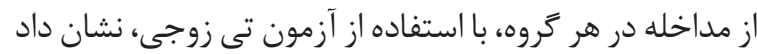

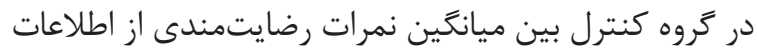

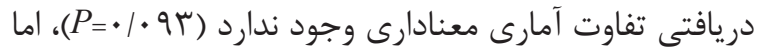

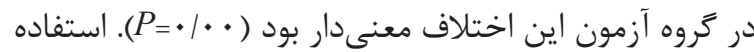

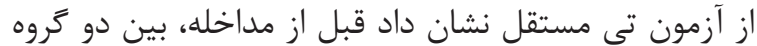

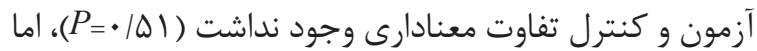

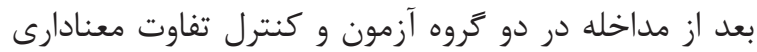

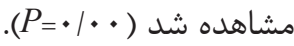

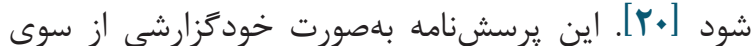

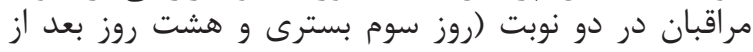

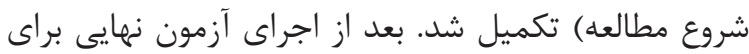

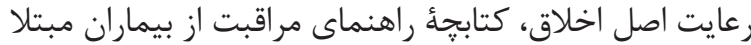

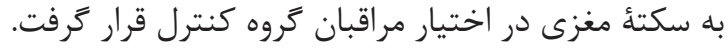

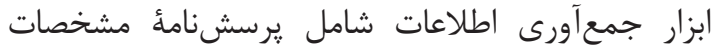

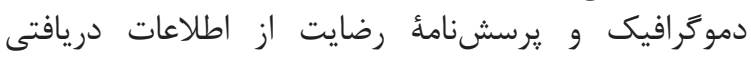

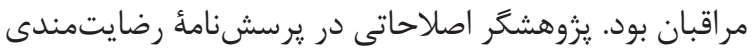

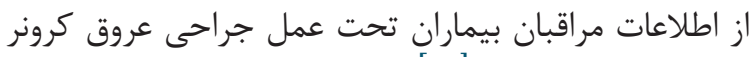

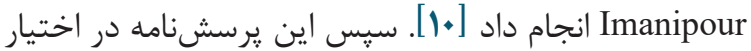

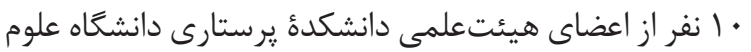

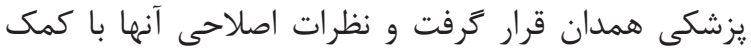

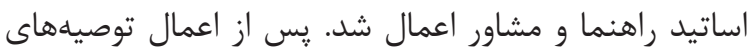

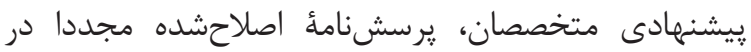

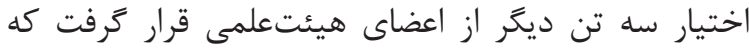

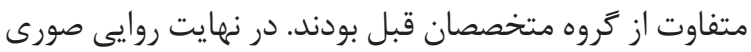

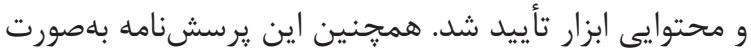

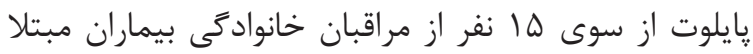

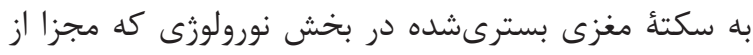

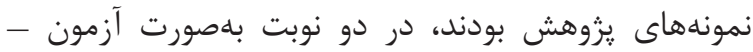

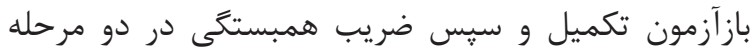

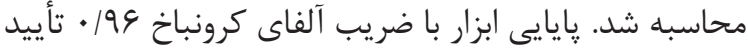
شده است. سته

يرسشنامه رضايت از اطلاعات دريافتى در بيماران سكتئ

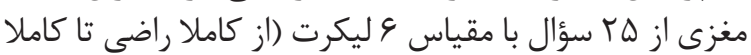

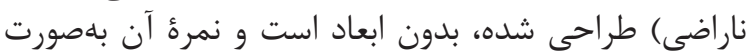

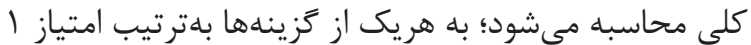

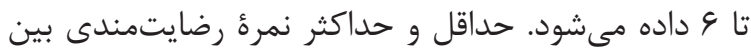

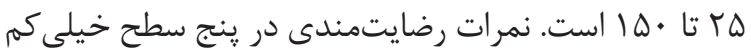

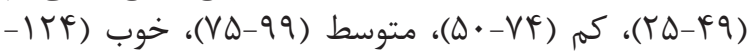

\begin{tabular}{|c|c|c|c|}
\hline \multirow{2}{*}{$P$ value } & \multicolumn{2}{|c|}{ 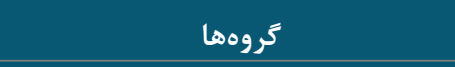 } & \multirow{2}{*}{ 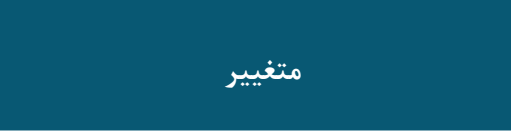 } \\
\hline & مداخله & 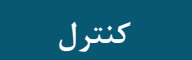 & \\
\hline$\cdot|\Delta|$ & $r / / \cdot \cdot \pm v q / 1 \Delta$ & $r G / T Y \pm \lambda r / V \Delta$ & رضايتمندى از حمايت اطلاعاتى قبل از مداخله \\
\hline \multirow[t]{2}{*}{$\cdot$} & $q / q \uparrow \pm|r| / \uparrow$ & $r V / r q \pm \Lambda q / \Delta \Lambda$ & رضايتمندى از حمايت اطلاعاتى بعد از مداخله \\
\hline & $\bullet /$. & .1 .94 & $P$ value \\
\hline
\end{tabular}

مداخله بهطور معنادارى نسبت به گروه كنترل افزايش يافته

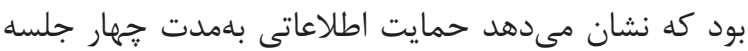

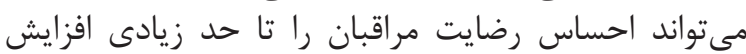

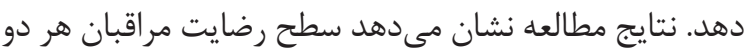

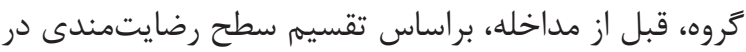

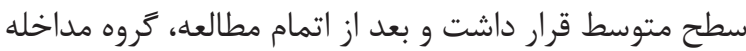

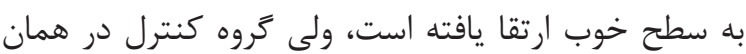
سطح متوسط رضايتمندى باقى ماند.

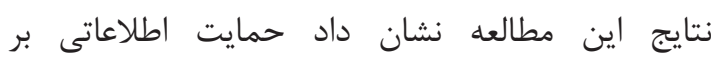

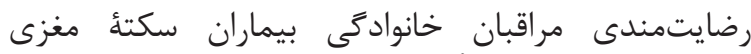

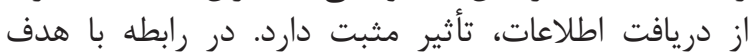

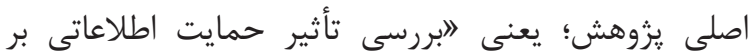

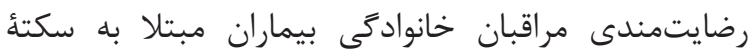

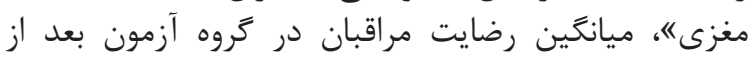




\section{نتيجهلَيرى}

نتايج مطالعه نشان ميدهدي حمايت اطلاعاتى از مراقبان

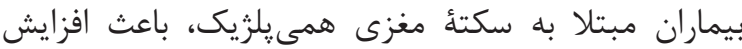

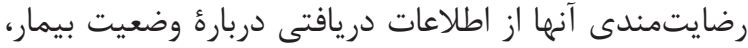

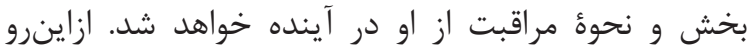

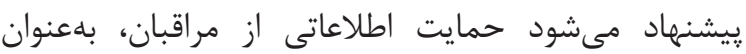

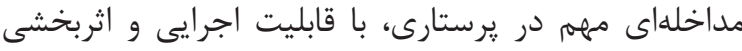

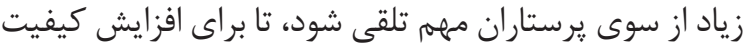

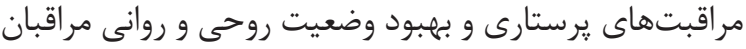
بيماران بهعنوان كروهى فراموش شرده، كامى برداشتيته شود.

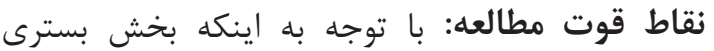

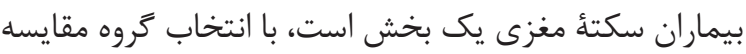

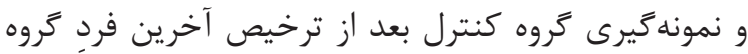

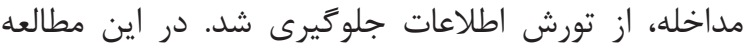

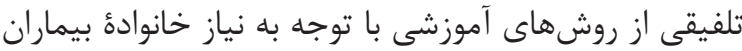

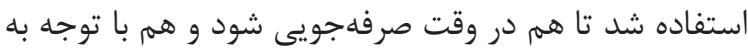

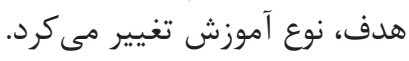

محدوديت مطالعه: با توجه به محدوديت زمانى مطالعها،

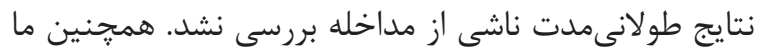

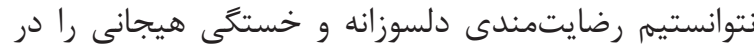

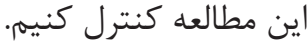

\section{سياسگزارى}

اين مقاله بخشى از طرح تحقيقاتى مصوب دانشخاه علوم

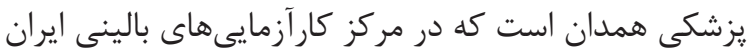

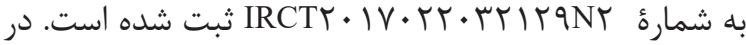

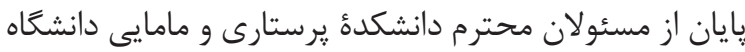

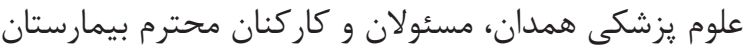

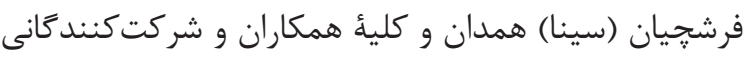

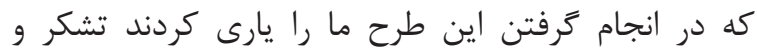

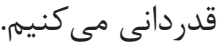

$$
\text { تعارض در منافع }
$$

بين نويسندًان هيجَّونه تعارضى در منافع وجود ندارد.
نتايج اين مطالعه با يافتههاى مطالعأ Kalra و همكاران

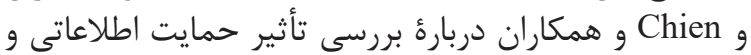

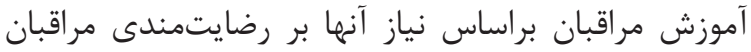

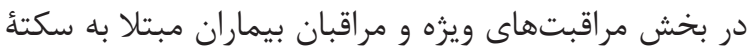

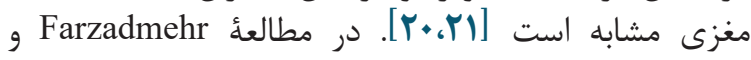

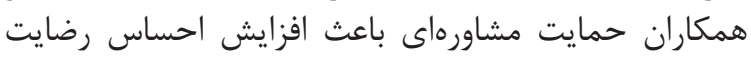

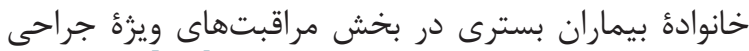

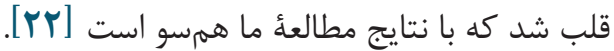

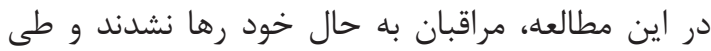

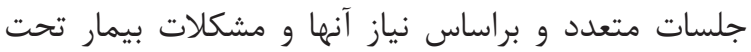

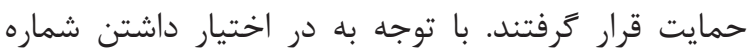

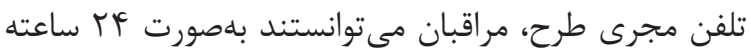

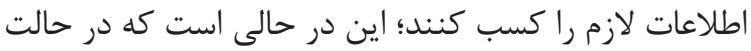

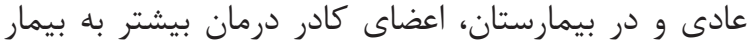

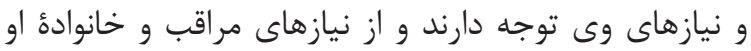

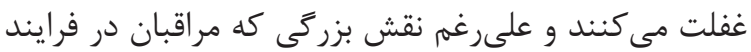

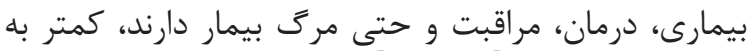

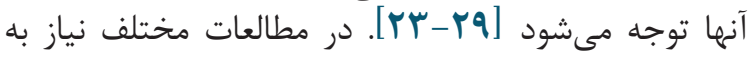

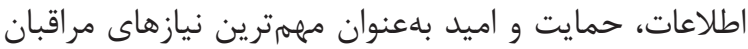

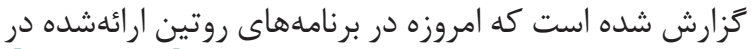

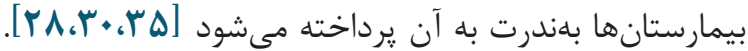

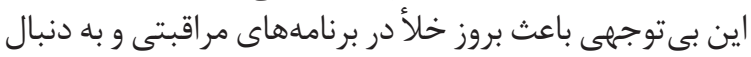

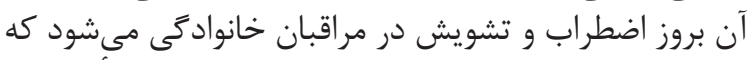

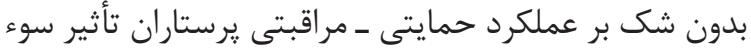

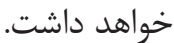

ييشنهاد براى مطالعات آينده: در مطالعات آتى لازم

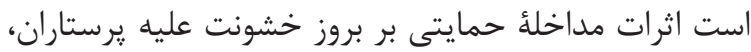

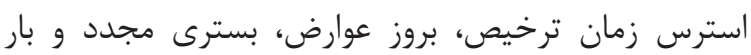

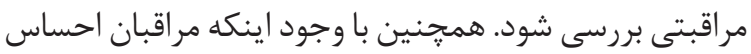

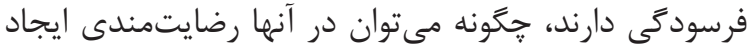

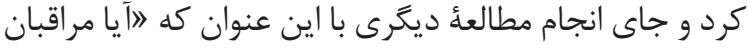

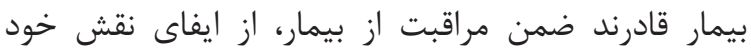

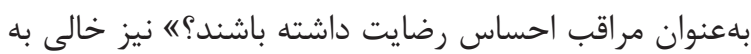
نظر مىرسد.

\section{References}

1. Karlsson C, Tisell A, Engstrom A, Andershed B. Family members' satisfaction with critical care: a pilot study. Nurs Crit Care. 2011;16(1):11-8. https://doi.org/10.1111/j.14785153.2010.00388.x PMID:21199550

2. Britton JR. Global satisfaction with perinatal hospital care: stability and relationship to anxiety, depression, and stressful medical events. Am J Med Qual. 2006;21(3):200-5. https://doi.org/10.1177/1062860606287191 PMID: $\underline{16679440}$

3. Miller YD, Dane AC, Thompson R. A call for better care: the impact of postnatal contact services

on women's parenting confidence and experiences of postpartum care in Queensland, Australia. BMC health services research. 2014;14(1):635. https://doi.org/10.1186/s 12913-014-0635-9 PMID:25526987 PMCID:PMC4301854

4. Jlala HA, Caljouw MA, Bedforth NM, Hardman JG. Patient satisfaction with perioperative care among patients having orthopedic surgery in a university hospital. Local and regional anesthesia. 2010;3:49. https://doi.org/10.2147/LRA. S11381 PMID:22915869

5. Monazea EM, Al-Attar GS. Quality of delivery care in Assiut University Hospital, Egypt: moth-

$$
\text { مجلئ علمى دانشكدة يرستارى و مامايى همدان }
$$


ers'satisfaction. Journal of the Egyptian Public Health Association. 2015;90(2):64-71. https:// doi.org/10.1097/01.EPX.0000466380.29269.4b PMID: $\underline{26154833}$

6. Gungor I, Beji NK. Development and psychometric testing of the scales for measuring maternal satisfaction in normal and caesarean birth. Midwifery. 2012;28(3):348-57. https://doi.org/10.1016/j. midw.2011.03.009 PMID:21546142

7. Crow R, Gage H, Hampson S, Hart J, Kimber A, Storey L, et al. The measurement of satisfaction with healthcare: implications for practice from a systematic review of the literature. Health Technol Assess. 2002;6(32):1-244. https://doi. org/10.3310/hta6320 PMID: 12925269

8. Rodgers H, Bond S, Curless R. Inadequacies in the provision of information to stroke patients and their families. Age and ageing. 2001;30(2):12933. https://doi.org/10.1093/ageing/30.2.129 PMID: 11395342

9. Minoli S, Kauer I, Colson V, Party V, Renou M, Anderson P, et al. Brief exposure to sensory cues elicits stimulus-nonspecific general sensitization in an insect. PLoS One. 2012;7(3):e34141. PMCID:PMC3311575

10. Imanipour M, Heidari Z, Seyedfatemi N, Haghani H. Effectiveness of Informational Support on Satisfication among Family Carers of Patients Undergone Open Heart Surgery. Journal of hayat. 2012;18(3):33-43.

11. Zhong M, Niu W, Lu ZJ, Sarov M, Murray JI, Janette J, et al. Genome-wide identification of binding sites defines distinct functions for Caenorhabditis elegans PHA-4/FOXA in development and environmental response. PLoS Genet. 2010;6(2):e1000848.PMCID:PMC2824807

12. Norouzinia R, Aghabarari M, Shiri M, Karimi M, Samami E. Communication barriers perceived by nurses and patients. Global journal of health science. 2016;8(6):65. https://doi.org/10.5539/gjhs. v8n6p65 PMID:26755475

13. Drummond A, Lincoln N, Juby L. Effects of a stroke unit on knowledge of stroke and experiences in hospital. Health Trends. 1996;28(1):2630 .

14. Knight K, Worrall L, Rose T. The provision of health information to stroke patients within an acute hospital setting: what actually happens and how do patients feel about it? Top Stroke Rehabil. 2006;13(1):78-98. https://doi.org/10.1310/ FC6M-P7L0-W3XD-4WAE PMID: 16581633

15. Cebeci F, Celik SS. Effects of discharge teaching and counselling on anxiety and depression level of CABG patients. Turkish J Thorac Cardiovasc Surg. 2011;19(2):170-6. https://doi.org/10.5606/
9اY. تأثير حمايت اطلاعاتى بر احساس رضايت مراقبان خانوادگى...

tgkdc.dergisi.2011.007

16. Siddiqui S, Sheikh F, Kamal R. What families want-an assessment of family expectations in the ICU. International archives of medicine. 2011;4(1):21. PMID:21696595

17. Kelson MC. Consumer collaboration, patient-defined outcomes and the preparation of Cochrane Reviews. Health Expect. 1999;2(2):129-35. PMCID:PMC5061448

18. Wellwood I, Dennis MS, Warlow CP. Perceptions and knowledge of stroke among surviving patients with stroke and their carers. Age and ageing. 1994;23(4):293-8. https://doi.org/10.1093/ ageing/23.4.293 PMID:

19. Burns N, Grove SK. The practice of nursing research. Conduct, critique \& utilization. 1993;4(5).

20. Kalra L, Evans A, Perez I, Melbourn A, Patel A, Knapp M, et al. Training carers of stroke patients: randomised controlled trial. BMJ. 2004;328(7448):1099. https://doi.org/10.1136/ bmj.328.7448.1099 PMID:15130977

21. Chien W-T, Chiu Y, Lam L-W, Ip W-Y. Effects of a needs-based education programme for family carers with a relative in an intensive care unit: a quasi-experimental study. International journal of nursing studies. 2006;43(1):39-50. https://doi.org/10.1016/j.ijnurstu.2005.01.006 PMID:16183062

22. Farzadmehr MA, Fallahi Khoshknab MA, Khankeh HR. The effect of nursing consultation on anxiety and satisfcation of patient's family in cardiac surgical intensive care unit. Journal of Nursing Education. 2016;4(2):57-64.

23. Dehghan Nayeri N, Mohammadi S, Pedram Razi S, Kazemnejad A. Adherence of Family Caregivers of Patients with Stroke to Rehabilitation Regimen. Hayat. 2012;18(1):30-41.

24. Hafsteinsdóttir TB, Vergunst M, Lindeman E, Schuurmans M. Educational needs of patients with a stroke and their caregivers: a systematic review of the literature. Patient education and counseling. 2011;85(1):14-25. https://doi. org/10.1016/j.pec.2010.07.046 PMID:20869189

25. Kazemnejad A. Effectiveness of family empowerment program on level of adherence to treatment regimens in stroke patients; a randomized controlled trial. Evidence Based Care. 2015;5(1):57-66.

26. Mitnick S, Leffler C, Hood VL, American College of Physicians Ethics P, Human Rights C. Family caregivers, patients and physicians: ethical guidance to optimize relationships. J Gen Intern Med. 2010;25(3):255-60. https://doi.org/10.1007/ s11606-009-1206-3 PMID:20063128 
27. Rabiei L, Mostafavi F, Masoudi R, Hassanzadeh A. The effect of family-based intervention on empowerment of the elders. Journal of education and health promotion. 2013;2.

28. Tsai PC, Yip PK, Tai JJ, Lou MF. Needs of family caregivers of stroke patients: a longitudinal study of caregivers' perspectives. Patient Prefer Adherence. 2015;9:449-57.PMID:25834409 PMCID:PMC4370911

29. Van Veenendaal H, Grinspun DR, Adriaanse HP. Educational needs of stroke survivors and their family members, as perceived by themselves and by health professionals. Patient Education and Counseling. 1996;28(3):265-76. https://doi. org/10.1016/0738-3991(95)00853-5

30. Mulhinch SF. Informal ALS caregivers: The relationship between coping strategies, resilience, and caregiver burden. Northcentral University: Northcentral University; 2016.

31. Lutz BJ, Young ME, Cox KJ, Martz C, Creasy KR. The crisis of stroke: experiences of patients and their family caregivers. Topics in stroke rehabilitation. 2015.
32. Chuluunbaatar E, Chou Y-J, Pu C. Quality of Life of Stroke Survivors and Their Informal Caregivers: A Prospective Study. Disability and Health Journal. 2015. PMID:27017120

33. Baumann M, Lurbe-Puerto K, Alzahouri K, Aïach P. Increased residual disability among poststroke survivors and the repercussions for the lives of informal caregivers. Topics in stroke rehabilitation. 2015.

34. Bagherbeik Tabrizi L, Navab E, Farokhnezhad Afshar P, Asadi Noghabi AA, Haghani H. Effect of Cognitive-Behavioral Intervention on Burden of Family Caregivers of Patients with Alzheimer's Disease. Journal of hayat. 2015;21(1):94102.

35. Salmani N, Ashketorab T, Hasanvand S. The Burden of Caregiverand Related Factorsof Oncology. Advances in Nursing \& Midwifery. 2014;24(84):11-8. 
1/.T. تأثير حمايت اطلاعاتى بر احساس رضايت مراقبان خانوادگى... 